\title{
Characterization of strain-induced martensite phase in austenitic stainless steel using a magnetic minor-loop scaling relation
}

\author{
Satoru Kobayashi, ${ }^{a)}$ Atsushi Saito, Seiki Takahashi, Yasuhiro Kamada, and \\ Hiroaki Kikuchi \\ NDE and Science Research Center, Faculty of Engineering, Iwate University, Ueda 4-3-5, \\ Morioka 020-8551, Japan
}

(Received 31 January 2008; accepted 17 April 2008; published online 8 May 2008)

\begin{abstract}
We propose a combined magnetic method using a scaling power-law rule and initial permeability in magnetic minor hysteresis loops for characterization of ferromagnetic $\alpha^{\prime}$ martensites in austenitic stainless steel. The scaling power law between the hysteresis loss and remanence is universal, being independent of volume fraction of strain-induced $\alpha^{\prime}$ martensites. A coefficient of the power law largely decreases with volume fraction, while the initial permeability linearly increases, reflecting a change in the morphology and quantity of martensites, respectively. The present method is highly effective for integrity assessment of austenitic stainless steels because of the sensitivity and extremely low measurement field. (C) 2008 American Institute of Physics.
\end{abstract}

[DOI: $10.1063 / 1.2924305]$

Austenitic stainless steels have been widely used for the manufacture of power plant structural components because of their high ductility, high corrosion resistance, high strength, etc. When subjected to external factors such as stress, fatigue, radiation, and creep, various types of lattice defects are induced. In particular, understress and dislocations are produced and piled up, and cracks are formed when the dislocation density locally maximizes. For 304 stainless steels with a metastable austenitic matrix, this degradation is always associated with martensite transformation. The accurate characterization of martensites therefore plays a key role in the integrity assessment of power plant components during operation.

For 304 stainless steels, plastic deformation at room temperature induces a transformation from a paramagnetic fcc austenite $(\gamma)$ to a ferromagnetic bcc martensite $\left(\alpha^{\prime}\right) .^{1-3}$ Since $\alpha^{\prime}$ martensite is the only ferromagnetic phase in the steel, a magnetic method to detect the initial permeability and/or saturation magnetization has been traditionally used to estimate the volume fraction of $\alpha^{\prime}$ martensites in the austenitic matrix. Recently, other magnetic hysteresis properties such as coercive force and remanence have been extensively investigated because they also provide useful information about the martensite transformation. ${ }^{4-10}$ It was revealed for plastically deformed 304 stainless steels that the coercive force $H_{c}$ depends on the volume fraction and decreases with increasing volume fraction, ${ }^{6-10}$ whereas some studies reported an increase. ${ }^{4,5}$ This behavior can be due to the interaction of the magnetic domain walls with the $\alpha^{\prime}-\gamma$ boundaries and the magnetostatic interaction between $\alpha^{\prime}$ martensite particles. Therefore, the coercive force is an indicator of the size and distribution of ferromagnetic $\alpha^{\prime}$ martensites formed in a paramagnetic austenitic matrix. However, high magnetic fields of the order of $1 \mathrm{MA} / \mathrm{m}$ are required to obtain the values of these properties. This is disadvantageous for the in situ integrity assessment that requires a compact measurement device.

\footnotetext{
${ }^{a)}$ Electronic mail: koba@iwate-u.ac.jp.
}

On the other hand, for ferritic steels, a relationship between the minor-loop flux density $B_{a}^{*}$ and minor-loop hysteresis loss $W_{F}^{*}$, obtained at the low and medium field range, has been used to evaluate the quality of steels. ${ }^{11}$ This relation, known as the Steinmetz law, ${ }^{12}$ is given by

$$
W_{F}^{*}=W_{F}^{0}\left(\frac{B_{a}^{*}}{B_{s}}\right)^{n_{F}},
$$

where $n_{F} \simeq 1.5$ and $W_{F}^{0}$ is a coefficient sensitive to the defect density and grain size. Our recent measurements on ferromagnetic materials ${ }^{13,14}$ have shown that $n_{F}$ is universally independent of the applied stress and temperature. It was found that $W_{F}^{0}$ is proportional to $H_{c}$ and can be obtained with extremely low applied fields, typically less than $4 \mathrm{kA} / \mathrm{m}$.

In this paper, we have examined magnetic minor hysteresis loops for 304 stainless steels after plastic deformation in tension. Unlike ferritic steels, 304 stainless steel includes a paramagnetic phase due to the austenitic matrix. In addition, $\alpha^{\prime}$ martensites are magnetically hard compared to ferritic steels. Therefore, the observed hysteresis loops are affected by the paramagnetic austenitic matrix and applied fields in addition to the ferromagnetic $\alpha^{\prime}$ martensites, as shown in Fig. 1(a). The observed magnetic flux density $B_{\text {obs }}$ is a summation of $\mu_{0} M_{m}, \mu_{a} H$, and $\mu_{0} H$ due to the $\alpha^{\prime}$ martensites, austenitic matrix, and applied field, respectively, where $\mu_{0}$ and $\mu_{a}$ denote the permeability of vacuum and the austenitic matrix, respectively. Consequently, properties of the $B_{\text {obs }}-H$ loop such as $B_{a}^{*}$ and the minor-loop coercive force $H_{c}^{*}$ are largely different from those of $\mu_{0} M_{m}-H$ loops, and the Steinmetz law will no longer hold true. In the present study, we propose another universal scaling rule in the minor hysteresis loops from which a magnetic property that is only sensitive to the $\alpha^{\prime}$ martensites can be obtained.

The chemical composition of 304 stainless steels used in this study is listed in Table I. Steel slabs with a thickness of $5 \mathrm{~mm}$ were austenitized at $1050{ }^{\circ} \mathrm{C}$ for $1 \mathrm{~h}$, followed by quenching using water. The slabs were then plastically deformed in tension at room temperature, with various levels of tensile stress up to $535 \mathrm{MPa}$. For magnetic measurements, we prepared two types of samples for each stress: rings 

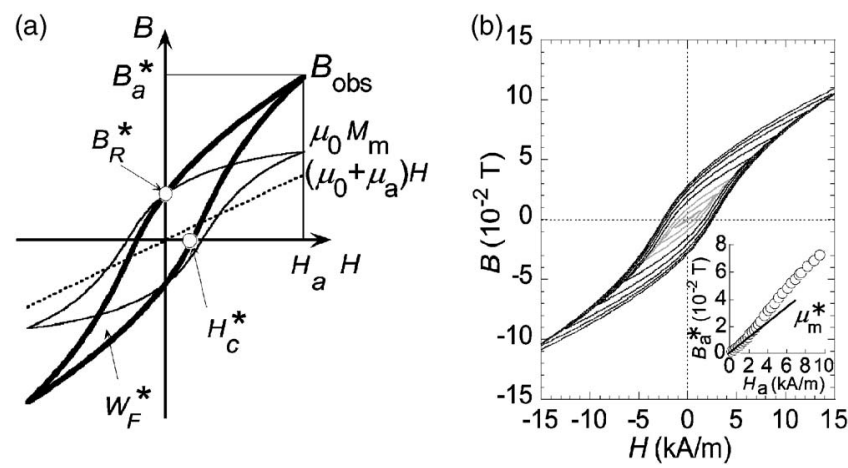

FIG. 1. (a) Schematic diagram of the hysteresis loops and the parameters of the $B_{\mathrm{obs}}-H$ loop. In addition to $\mu_{0} M_{m}$ (thin line) due to ferromagnetic $\alpha^{\prime}$ martensites, both $\mu_{a} H$ and $\mu_{0} H$ (dotted line) are involved in the $B_{\mathrm{obs}}-H$ (thick line) loop. (b) A set of minor hysteresis loops. The data for $502 \mathrm{MPa}$ are given as an example. Minor loops with $H_{a}$ below $4 \mathrm{kA} / \mathrm{m}$ (gray loops) were used to obtain $W_{m}^{0}$. The inset shows $B_{a}^{*}$ as a function of $H_{a} \cdot \mu_{m}^{*}$ was obtained from a linear part of the $B_{a}^{*}-H_{a}$ curve below $H_{a}=1 \mathrm{kA} / \mathrm{m}$.

wound with 220-turn exciting and 120-turn pickup coils and small plates with the long axis parallel to the stress direction. The plates were used to determine the volume fraction of $\alpha^{\prime}$ martensites by saturation magnetization measurements using a superconducting quantum interference device (SQUID) magnetometer (Quantum Design MPMS-XL). A saturation magnetization of $154 \mathrm{emu} / \mathrm{g}$ was assumed for a volume fraction of $100 \%$. As shown in the inset in Fig. 2(a), the volume fraction of $\alpha^{\prime}$ martensites increases with stress and reaches $19.5 \%$ after plastic deformation by the maximum stress of $535 \mathrm{MPa}$. A set of magnetic minor hysteresis loops with various field amplitudes $H_{a}$ up to $20 \mathrm{kA} / \mathrm{m}$ was measured for the ring samples using a fluxmeter. A triangular current with a frequency of $0.2 \mathrm{~Hz}$ was applied to the exciting coil to generate a cyclic magnetic field and magnetize the sample. The magnetic field within the sample was obtained from the voltage across a $1-\Omega$ resistance connected to the exciting coil in series. The output signals of the pickup coil were amplified and then purified by a low-pass filter with a cutoff frequency of $40 \mathrm{~Hz}$. The signals were integrated in order to obtain the magnetic flux within the sample. Figure 1(b) shows an example of a set of minor loops that we measured. A cyclic magnetic field with $H_{a}$ was applied in a demagnetized state and a minor loop, which is symmetric about the origin, was measured. By step-by-step increasing $H_{a}$, a set of minor loops was obtained. These minor loops are different from the major loop obtained with $H_{a}$ that is sufficiently large for saturation.

For each minor loop with different $H_{a}$, the parameters denoted in Fig. 1(a) were obtained and the relations between them were examined. Figure 2(a) shows a double logarithmic plot of a relation between $W_{F}^{*}$ and $B_{a}^{*}$ taken for various levels of stress. Unlike ferritic steels, no linear part was detected over the entire range of $B_{a}^{*}$. The observation shows that the Steinmetz law, given by Eq. (1), does not hold true for deformed 304 stainless steels where ferromagnetic $\alpha^{\prime}$ martensites are induced. This is attributed to the fact that the para-

TABLE I. Chemical composition of 304 stainless steels.

\begin{tabular}{ccccccccc}
\hline \hline Element & $\mathrm{C}$ & $\mathrm{Si}$ & $\mathrm{Mn}$ & $\mathrm{P}$ & $\mathrm{S}$ & $\mathrm{Ni}$ & $\mathrm{Cr}$ & $\mathrm{Fe}$ \\
\hline wt \% & 0.088 & 0.480 & 1.630 & 0.023 & 0.001 & 8.200 & 18.700 & bal.
\end{tabular}
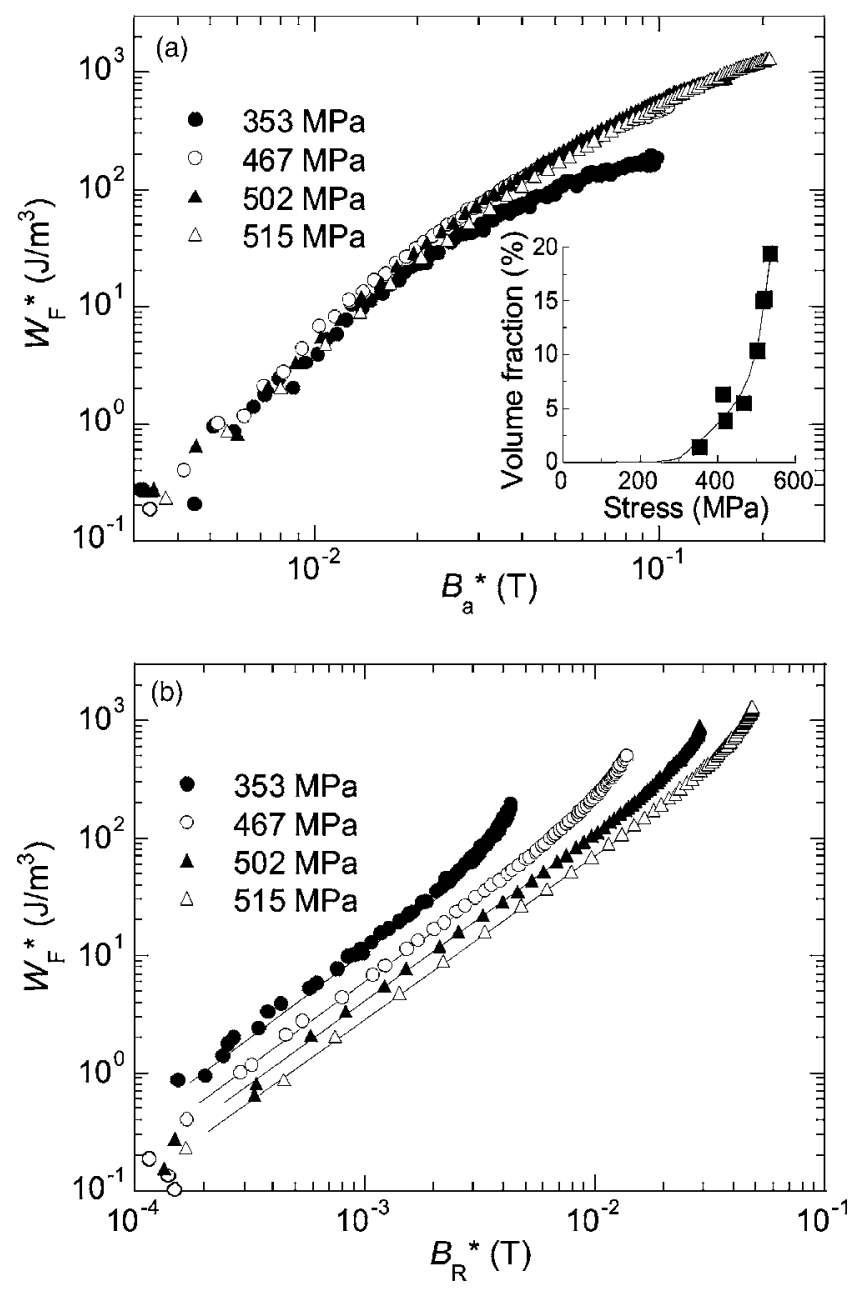

FIG. 2. The relations (a) between $W_{F}^{*}$ and $B_{a}^{*}$ and (b) between $W_{F}^{*}$ and $B_{R}^{*}$ plotted on a double logarithmic scale. The solid lines in (b) denote the least-squares fitting lines. The inset in (a) shows the volume fraction of $\alpha^{\prime}$ martensites as a function of the tensile stress.

magnetic austenite matrix and applied field are also involved in the value of $B_{a}^{*}$.

On the other hand, the two minor-loop parameters, namely the minor-loop remanence $B_{R}^{*}$ and $W_{F}^{*}$ only reflect the ferromagnetic $\alpha^{\prime}$ martensites and are not affected by the austenitic matrix and applied fields. Therefore, a magnetic property obtained from the relation between these two parameters may reveal the intrinsic features of $\alpha^{\prime}$ martensites. Figure 2(b) shows the relation plotted on a double logarithmic scale for various levels of stress. In contrast to the $W_{F}^{*}-B_{a}^{*}$ curves shown in Fig. 2(a), the $W_{F}^{*}-B_{R}^{*}$ curves exhibit straight lines over a wide range of $W_{F}^{*}$ and $B_{R}^{*}$. This range shifts toward higher values with increasing applied stress because both parameters are proportional to the volume fraction of $\alpha^{\prime}$ martensites. This linearity strongly indicates the presence of a scaling power-law rule between $W_{F}^{*}$ and $B_{R}^{*}$. In order to extract the intrinsic magnetic properties of $\alpha^{\prime}$ martensites, we assume an equation given as

$$
W_{F}^{*}=W_{m}^{0}\left(\frac{B_{R}^{*}}{B_{R}}\right)^{n_{m}},
$$

where $W_{m}^{0}$ and $n_{m}$ are a coefficient and power-law exponent, respectively. Least-squares fits to Eq. (2) yielded an almost constant value of $n_{m}=1.40 \pm 0.04$. Here, minor loops with $H_{a}$ below $4 \mathrm{kA} / \mathrm{m}$ were used for the fits. This value is indepenicense or copyright, see http://apl.aip.org/apl/copyright.jsp 


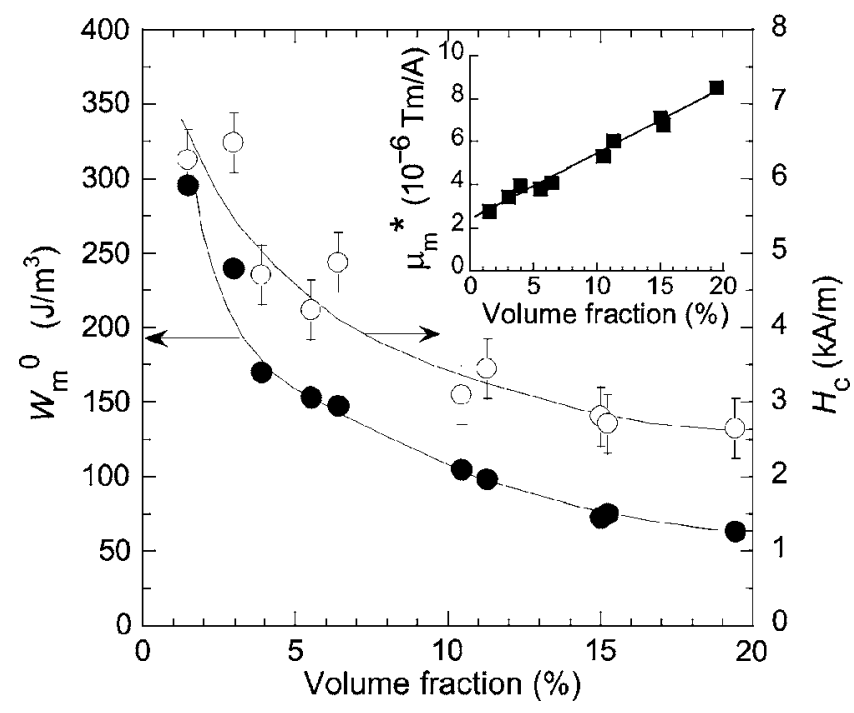

FIG. 3. Minor-loop coefficient $W_{m}^{0}$ as a function of the volume fraction of martensites. For comparison, $H_{c}$ obtained from the saturation magnetization measurements is also given.

dent of the volume fraction of $\alpha^{\prime}$ martensites, strongly indicating the presence of a universal scaling power-law rule for strain-induced ferromagnetic $\alpha^{\prime}$ martensites.

Figure 3 shows $W_{m}^{0}$ as a function of the volume fraction of $\alpha^{\prime}$ martensites. $W_{m}^{0}$ sharply decreases with increasing volume fraction below $3 \%$ and then slowly decreases up to a maximum volume fraction of $19.5 \%$. This trend is similar to that of $H_{c}$ obtained from the saturation magnetization measurements. However, the decrease in $W_{m}^{0}$ from $1.5 \%$ to $19.5 \%$ fraction is approximately $80 \%$ and much larger than $58 \%$ in the case of $H_{c}$. This indicates the high sensitivity of $W_{m}^{0}$ to ferromagnetic $\alpha^{\prime}$ martensites.

During plastic deformation, an austenitic structure transforms into $\alpha^{\prime}$ martensites by shearing the lattice in order to lower the stacking fault energy associated with increasing dislocation density. At the initial stage of plastic deformation, small $\alpha^{\prime}$ martensite particles are nucleated around the intersection of the shear bands. ${ }^{1-3}$ As the plastic deformation progresses, $\alpha^{\prime}$ martensite particles grow, merge with each other, and form larger clusters. When the volume fraction is low, $\alpha^{\prime}$ martensite particles (clusters) can be considered to be isolated ones with no interparticle interactions. In this case, $H_{c}$ would be mainly determined by the size ${ }^{7,9,10}$ because the pinning strength of the magnetic domain walls at the $\alpha^{\prime}-\gamma$ boundaries weakens with the volume-to-surface ratio. The decrease in $H_{c}$ with increasing volume fraction therefore reflects an increase in the particle (cluster) size. Since $W_{m}^{0}$ has a good proportional relation with $H_{c}, W_{m}^{0}$ is a good indicator of the size of $\alpha^{\prime}$ martensites.

It should be noted that some studies using a fluxmeter (ac measurements) reported an increase in $H_{c}$ with the volume fraction. ${ }^{4,5}$ This may be attributed to the fact that the effects of magnetic fields $\mu_{0} H$ on the hysteresis loops were not considered in the analysis. In fact, all dc measurements using instruments such as a SQUID magnetometer ${ }^{8-10}$ and vibrating sample magnetometer, ${ }^{6,7}$ yielded a decrease in $H_{c}$ with volume fraction.
The minor-loop coefficient $W_{m}^{0}$ is a structure-sensitive magnetic property similar to $W_{F}^{0}$ obtained from the Steinmetz law. $W_{m}^{0}$ provides useful information about the size of $\alpha^{\prime}$ martensite particles. However, it does not depend on the quantity of $\alpha^{\prime}$ martensites itself. This is because both $W_{F}^{*}$ and $B_{R}^{*}$ are parameters that depend on their quantity ${ }^{4}$ and the scaling treatment of Eq. (2) makes $W_{m}^{0}$ independent. Nevertheless, by measuring the initial permeability obtained from a set of minor hysteresis loops, the volume fraction of $\alpha^{\prime}$ martensites can be easily estimated. Here, the initial permeability is mainly dominated by the reversible motion of domain walls within $\alpha^{\prime}$ martensite particles and is generally proportional to the saturation magnetization of the material. ${ }^{11}$ The inset in Fig. 3 shows the minor-loop initial permeability $\mu_{m}^{*}$ as a function of the volume fraction. Here, $\mu_{m}^{*}$ was obtained from a linear part of the $B_{a}^{*}-H_{a}$ curves below $H_{a}=1 \mathrm{kA} / \mathrm{m}$, as shown in the inset in Fig. 1(b). It is clearly observed that $\mu_{m}^{*}$ is linearly proportional to the volume fraction. Therefore, by measuring $\mu_{m}^{*}$ as well as $W_{m}^{0}$, it is possible to simultaneously obtain information about both the volume fraction and morphology of $\alpha^{\prime}$ martensites.

In conclusion, a universal scaling power-law rule between the hysteresis loss and the remanence of minor hysteresis loops was found for stain-induced ferromagnetic $\alpha^{\prime}$ martensites in 304 stainless steels. A coefficient of the power law, which is sensitive to the size and distribution of $\alpha^{\prime}$ martensites, was obtained for low applied fields less than several $\mathrm{kA} / \mathrm{m}$. This magnetic method using minor hysteresis loops can be applied for the integrity assessment of austenitic stainless steel components in power plants. Finally, this method is also useful for the characterization of artificial magnetic materials where ferromagnetic nanoparticles are embedded in a non-magnetic or antiferromagnetic matrix.

This research was supported by a Grant-in-Aid for Scientific Research (S), Grant No. 14102034, from the Ministry of Education, Culture, Sports, Science and Technology of Japan.

${ }^{1}$ P. L. Mangonon and G. Thomas, Metall. Trans. 1, 1577 (1970).

${ }^{2}$ S. S. Hecker, M. G. Stout, K. P. Staudhammer, and J. L. Smith, Metall. Trans. A 13A, 619 (1982).

${ }^{3}$ R. P. Reed and C. J. Guntner, Trans. Metall. Soc. AIME 230, 1713 (1964). ${ }^{4}$ A. Mitra, P. K. Srivastava, P. K. De, D. K. Bhattacharya, and D. C. Jiles, Metall. Mater. Trans. A 35A, 599 (2004).

${ }^{5}$ I. Meszaros and J. Prohaszka, J. Mater. Process. Technol. 161, 162 (2005).

${ }^{6}$ J. Ding, H. Huang, P. G. McCormick, and R. Street, J. Magn. Magn. Mater. 139, 109 (1995).

${ }^{7}$ S. S. M. Tavares, M. R. da Silva, J. M. Neto, S. Miraglia, and D. Fruchart, J. Magn. Magn. Mater. 242-245, 1391 (2002).

${ }^{8}$ K. Mumtaz, S. Takahashi, J. Echigoya, Y. Kamada, L. Zhang, H. Kikuchi, K. Ara, and M. Sato, J. Mater. Sci. 39, 85 (2004).

${ }^{9}$ L. Zhang, S. Takahashi, Y. Kamada, H. Kikuchi, K. Ara, M. Sato, and T. Tsukada, J. Mater. Sci. 40, 2709 (2005).

${ }^{10}$ L. Zhang, S. Takahashi, and Y. Kamada, Scr. Mater. 57, 711 (2007).

${ }^{11}$ R. M. Bozorth, Ferromagnetism (IEEE, New York, 1978).

${ }^{12}$ C. P. Steinmetz, Trans. Am. Inst. Electr. Eng. 9, 3 (1892).

${ }^{13}$ S. Kobayashi, S. Takahashi, Y. Kamada, H. Kikuchi, and K. Ara, J. Appl. Phys. 99, 08H908 (2006).

${ }^{14}$ S. Kobayashi, T. Fujiwara, M. Tsunoda, S. Takahashi, H. Kikuchi, Y. Kamada, K. Ara, and T. Shishido, J. Magn. Magn. Mater. 310, 2638 (2007). 